\title{
The Positioning of Interpreting and Interpreters From the Perspective of the Otherness of Understanding*
}

\author{
REN Rui, ZHENG Ya-li, LI Yang \\ Northeastern University, Shenyang, China
}

\begin{abstract}
Interpreter is sure to be the subject of interpreting. In the activity of interpreting, interpreter is the doer, while the audience is the receiver of the interpreted content. Otherness is a term borrowed from philosophy, which is closely related to self and the other. In interpreting, there is a self of the interpreter and the other of the audience. And the neutrality or invisibility of interpreters is relative. Factually, interpreter is the true controller of the cross-cultural communication. In the world of interpreting, the positioning of interpreting and interpreters is doomed to be a tilt balance. The tilt is determined by the otherness of understanding. Consequently, interpreting is other-oriented, namely, audience-centered interpreting.
\end{abstract}

Keywords: interpreting, interpreter, positioning, otherness, understanding

\section{Introduction}

Interpreting is commonly depicted as "a purposeful message-sending from the source to the target language" (REN, 2014, p. 2). In this process of message-sending, information is finally transferred from one language to another and from speaker to audience through the proper interpretation by the interpreter. Usually, we focus on facts like "the interpreted communicative event (ICE)" (Angelelli, 2004b, p. 8) involves three parties that participate in cross-cultural communication: both sides of the conversation and interpreter. In cross-cultural communication in which the interpreter is involved, the real leading role lies in interpreter. The speaker, no matter how important he or she is, the ultimate talk in the target language is between interpreter and the audience. Thus, in the community of interpreting, speaker could be secondary or ignored though the source language is vital. The self of interpreter and the other of the audience make the final community. Only by achieving the understanding between self and the other, can we say the ICE is successful lastly. Interpreter's interpreting is an activity of solving cross-cultural riddles (REN, 2014, p. 8). In the process of riddle-reading, there is a highly complex procedure involving information processing between language and culture, as well as the account of social factors. Whether understanding could be achieved is not determined by interpreter but the audience. In search of a successful interpreting, interpreters should not only focus on analysis, conversion, and expression of

\footnotetext{
* Acknowledgements: This research is funded by Education Department, Liaoning Province, China. Project of Humanities and Social Sciences (No. W2012038), which is headed by Associate Professor REN Rui.

REN Rui, associate professor, master, English Department, Northeastern University. Doctor candidate of Foreign Philosophy at School of Philosophy and Society, Jilin University.

ZHENG Ya-li, postgraduate, English Department, Northeastern University.

LI Yang, undergraduate, English Department, Northeastern University.
} 
the target language, but also take the target audience and their needs into account. Therefore, interpreters need to know about the audience type and the knowledge levels, levels of acceptance of target audience so as to make timely adjustments to their own interpreting strategies according to the response and feedback by audience. The visibility of interpreters is no more a secret in the long debate over the presence of in interpreting (Angelelli, 2004a, pp. 3-4; Angelelli, 2004b, pp. 8-9; REN, 2009, pp. 540-544, 555). Since interpreter could be the controller of the interpretation, interpreter could take one side of the two parties of the speaker and the audience, for instance, in medical interpreting (Angelelli, 2004b, p. 75). Depending on the technicality of a speech, interpreter might explain professional knowledge related to the speech or domestic cultural and religious traditions may be considered in interpreting. All in all, sometimes interpreters need to explain and supplement the source language so as to comprehensively convey the speaker's message and ensure good communication between the speaker and the audience. For example, interpreters need to explain things like omitted part of the speech given by speaker, metaphor use, and words with specific cultural connotations. Therefore, necessary explanations and supplements by interpreters in interpreting could significantly better audience's understanding the source language.

\section{Self, the Other, and Otherness in Philosophy}

There has been a long tradition of researching the other in philosophy. The other is a concept closely related to self. According to the Wikipedia (2015), the concept that the Self requires the existence of the other, as the counterpart who defines the Self. Hegel was said to be among the first to have introduced the idea of the other as constituent in self-consciousness. The other is a concept of the identity of difference, and the other/other is also taken as dissimilar or opposite to being "us" or the Same. The explanation of "otherness" was found under the term of alterity. Alterity is a philosophical and anthropological term meaning "otherness", strictly being in the sense of the other of two (Latin alter). The terms the "other", "other", and "otherness" refer to who and what is distinct or separate from identity, and from the Self.

\section{Otherness of Understanding in Interpreting Between Interpreter and the Audience}

There is otherness of understanding between any two persons. What is closely related to the otherness of understanding in interpreting are another two facts: certainty and uncertainty of understanding between interpreter and the audience.

Uncertainty of understanding is a fact being often neglected.

Indeed, language is used by self.

In this case, people tend to put themselves in the center of language communications and take it for granted that since language is used by us, then listener will surely or definitely understand what I mean. As a matter of fact, language communication can be carried out smoothly when speaker speaks, listener listens, and listener well understands speaker. This is a quite common phenomenon in language communications. But it does not necessarily be like this. We cannot say there is definitely no communication barriers in people-to-people exchange and understanding can always be achieved. Another phenomenon in language communication, in fact, is also an objective existence, which is when understanding fails to be achieved and problems like misunderstanding and non-understanding occur in the process. Understanding problems do not 
exist for self as an individual, because only one person is involved. But in a dialogue where two parties of communication are all involved, uncertainty of understanding become unavoidable. Whether the meaning speaker intends to express by language can appear in listener's mind, namely whether listener's comprehension is consistent with what the speaker wants to say, is indeed a problem with uncertainty. If speaker and listener fail to agree on the understanding of the same language in their minds, then there will be some understanding problems. It is worth noting that even in dialogues where both sides use the same language to communicate, misunderstanding and non-understanding occur very often (REN, 2006).

However, no matter how many barriers there might be between two persons (of one language or not) in understanding, certainty of understanding is also a matter of fact.

Differences in cross-cultural communication increase the possibility of understanding problems in interpretation. However, while we are elaborating on the nature of such understanding problems, we also need to be clear that understanding is an objective necessity. The premise of achieving understanding is the commonality of language between self and the other. The foothold of the language's privacy rests with the fact that language exists in the mind of individuals. Seemingly, each person's experience is his or her personal affairs so no other people can penetrate into my consciousness and share my experience. It is only I that can actually know where it hurts in my body. When we regard the language in a person's mind as an individual's personal feeling or inner experience, since no one can enter the individual's mind to feel or experience his thinking, under this circumstance, we are likely to believe that language is private. Wittgenstein illustrated that private language was impossible (Wittgenstein, 1953, p. 131).

Goldstein thought that,

The flexible use of language represents the human lifestyle, and it particularly shows the way of being with others. In the field of my language, the existence of others is without any doubt. In spite of my incomplete recognition of others, I still know they exist. Language, as an inner being, connects us with our peers and the dynamic relationship between self and the other is established by language. (REN, 2014, p. 11)

Therefore, it is common language, living in nature, which is used by people as a communicating tool. The language used in cross-cultural communication and interpreting is a common language between people; hence understanding can certainly be reached. The reason for understanding barriers is the existence of some problems. When these problems are successfully resolved, communication can be carried out smoothly. That is to say, the uncertainty of understanding does not necessarily mean that understanding problems cannot be dealt with. On the contrary, understanding is indeed an issue that can be solved (REN, 2014, p. 12).

Comprehension in the context of target language and the positioning of interpreting and interpreters can be discussed from the perspective of dialogue between interpreter and audience. Fundamentally, the cross-cultural communication is the exchange and understanding between the interpreter and the audience. Speaker, no matter how important he or she might be, only provides messages to be delivered for interpreter. But it is the audience that actually defines the whole interpreting activity and the positioning of interpreter. It has been proven that comprehension, both in single and multilingual communication, owns the feature of otherness. That means whether comprehension can be achieved or not is not determined by self, but the other. In other words, it is not the self's intention that counts, but the other's reception condition. In regard to interpretation, interpreter's understanding of the speaker's message only plays a secondary role in the positioning of interpreting and 
interpreter. As to the result of interpretation, understanding between interpreter and audience plays a decisive role in determining whether the interpretation is a success or a disaster, and whether the judgment for interpreter's performance is positive or negative.

Therefore, in this message-sending process, it is a subtraction other than an addition in terms of the number of communicative parties. In the reformulation stage of interpreting or in the target communication stage of interpreting, speaker becomes thingless or invisible. In interpreting, the three-party-communication of the speaker-interpreter-listener in interpreting is sure to reduce to two parties, namely interpreter and the audience, in the reformulation stage of interpreting. So we can get a tilt balance: audience $>$ speaker.

\section{The Tilt Balance of the Other Over Self: Audience-Centered Interpreting}

Seemingly, interpreting is an interpreter controlling world. As a matter of fact, language, meaning, and understanding are social events involving self and the other. Even when language has not been voiced out, meaning has not been expressed, and understanding has not been achieved, the other has already been there as the object. The other not only exists but takes precedence over self, namely the other over self. The being of language, meaning and understanding make the coexistence of self and the other (REN, 2009).

The interpreter's interpreting is to solve cross-cultural puzzles. It is true that interpreting process involving decoding, inference, and coding by interpreter, which is a highly complicated one. And language use is far more important than language performance itself in exchange activities. The cognitive, social, and cultural factors of language use play a more significant role in this process. Therefore, it has been decided that interpreter has to master the pragmatic features of two languages when interpreting two cultures.

Interpreting is a highly complex procedure involving information processing between language and culture, as well as the consideration for social factors. Interpreter should be both visible and invisible as far as interpreting is concerned. Being visible means interpreter needs to visibly resolve lingual, cultural, and social problems. While being invisible, interpreter is supposed to act as a bridge connecting two sides of the conversation and convey the exact meaning without distorting or changing the source language (REN, 2014, p. 93).

The most critical part in interpreting lies in the understanding of the interpreted content by the audience. Whether the meaning can be sunk in depends on the audience, rather than the interpreter. The relation between audience and interpreter is determined by otherness of understanding.

In the dialogue between self and the other, the understanding can be achieved and the dialogue can be continued only when the other's comprehension about self's linguistic meaning is consistent with the exact meaning the self wants to express. In such a dialogue, understanding is not about self, the speaker, but about the other, the listener. Therefore, "otherness of the understanding" makes the other real dominator in the dialogue (REN, 2014, p. 9).

When it comes to type of understanding problems, understanding, in people-to-people exchange, has always been taken for granted. However, when self and the other fail to agree on the meaning expressed by language, understanding problems like non-understanding and misunderstanding come naturally. "Non-understanding occurs when listener can only understand very limited speech or even understand nothing, and possibly when listener encounters somewhat sudden topic switch, which overwhelms him or her" (REN, 2006, p. 446). 
Misunderstanding means two sides of the speech "giving different meanings to the same linguistic unit". In this way, "when there is an illusion of partial or complete, temporary or permanent understanding between two sides, misunderstanding problem emerges." However, as far as listener is concerned, the possible condition is that "Listener develops a kind of arguable and reasonable understanding, as he or she might think so, but actually it's far from what the speaker wants to express" (REN, 2014, p. 9).

Audience can be regarded as a stimulus affecting interpreters' performance. There are two reasons. Firstly, audience's acceptance and understanding of the interpretation are the ultimate goals for interpreting activities. Secondly, the audience's words, hints, sighs, gestures, and expressions may all affect the quality of interpreter's work. This process can be well explained by Stimulus-Response Theory: Audience's specific response will have a direct or indirect impact on the interpreter's mentality and their interpreting strategies.

Interpreters should not only focus on analysis, conversion, and expression of the target language, but also take the target audience and their needs for consideration. Therefore, interpreters need to know about the audience type and know about their needs, knowledge level, level of acceptance, their ways of absorbing information, and their aesthetic standards. Only by knowing all these things can interpreters select appropriate interpreting methods. In order to give better interpreting performance, interpreters also need to think about audience's level of acceptance and make timely adjustments to their own strategies according to the response and feedback by audience. The interpreting activities are satisfactorily completed only when audience fully understand and accept the messages sent by the speaker (REN, 2014, p. 111).

\section{Audience-Centered Interpreting Strategies}

We can generally divide the audience in an interpreting activity into two types: ordinary audience and professional audience.

Ordinary audience refers to audience attending banquets, ceremonies, speeches, reports, and other similar foreign affair activities. They usually have the following features: They have lower demands for the professionalism of interpreter's language expression, so the interpreter is allowed to convey the original information with simple and colloquial words, rather than some specialized terms. They are inferior to professional audience in terms of level of expertise, so interpreter has more choices in selecting words and expressions they use in interpreting. Most ordinary audience do not understand the original language, and that is why they cannot either make comparisons between the target language and original language or give timely and accurate evaluation of interpreter's performance, which will greatly reduce the interpreter's psychological pressure in interpreting process. Most ordinary audience are not celebrities or VIPs (Very Important Persons), so the psychical distance between them and the interpreter is relatively negligible and it is far more easier and simpler for interpreters to communicate with them. In this way, interpreters are able to take it easy with their work. However, due to the lack of professional knowledge about the speech or report content in interpreting activities, coupled with differences in cultural background, ordinary audience tend to give different feedback to interpreters because of the hard-to-understand contents in the meetings (REN, 2014, p. 108).

As to professional audience, things could be much easier. Not all audience in interpreting activities is ordinary one without professional knowledge background. In occasions such as academic conferences, seminars, symposiums, negotiating meetings, workshops, and laboratories, the audience are mostly domestic or 
foreign scholars, CEOs (Chief Executive Officers) of companies and experts in that area. They are called professional audience. This kind of audience usually has extensive professional knowledge of culture, which makes them have a more comprehensive and profound understanding of the interpretation content than ordinary audience. Therefore, interpreters should take full consideration of the professional audience and their impacts. They usually have the following features: Professional audience have a more profound and thorough understanding of information in interpreting activities, so that they can make a more professional evaluation of the accuracy and the overall quality of interpreter's performance. They are usually equipped with rich professional knowledge, which makes it possible for them to supplement and correct some minor mistakes that occur in interpreting and turn to the source language when necessary for seeking direct understandings. They are familiar with relevant cultural background knowledge, and most of them might be working in international or multicultural environments. Most of professional audience can speak foreign language. This, on the one hand, helps to make them fully understand the information expressed by interpreters, but on the other hand, since they may immediately correct interpreters' mistakes, will also give interpreters tremendous psychological pressure (REN, 2014, p. 109).

\section{Conclusion}

In interpreting, interpreter is the very controller of the cross-cultural communication and should be both visible and invisible. In understanding, there is sure to be a tilt balance between self and the other, namely, self leaning to the other. To be specific, in interpreting, there is a tilt balance between interpreter and the audience, namely, interpreter leaning to the audience, or audience-centered interpreting.

"Translator is to be out of the original (or speaker's) culture and then enters into the culture of the target text (or audience)" (REN, 2014, p. 1). As a bridge and intermediary of cultural exchange, the essence of interpretation is communication. Interpreter, as a solver of cross-cultural language puzzle, needs to be fully aware of all factors that may bring about understanding problems in both two language cultures. Only in this way can interpreters play their due roles in connecting two language cultures by overcoming possible misunderstanding and non-understanding problems and ensuring smooth communication.

The self positioning of the interpreter plays a decisive role in the quality of interpreting activities. Correct positioning is the precondition of ensuring the quality of interpretation. Interpreter's role or positioning is closely related to what people think about interpreting activities, and in the various stages of interpretation research, people have put forward many ideas about the interpreter's presence. The presence positioning of interpreters is gradually changing over time. With the deepening of people's understanding of interpreting activities, the self positioning problems of interpreters have become more and more clear. The presence of interpreters mainly involves invisibility theory and visibility theory. Interpreter should be both visible and invisible when doing interpretation. Being visible means interpreter needs to resolve lingual, cultural, and social problems, while at the same time, interpreter is supposed to act as a bridge connecting two sides of the conversation and convey the exact meaning without distorting or changing the information, which is being invisible.

\section{References}

Alterity. (n.d.). In Wikipedia. Retrieved from https://en.wikipedia.org/wiki/Alterity

Angelelli, C. V. (2004a). Revisiting interpreter's role. Amsterdam: John Benjamins Publishing Company. 
Angelelli, C. V. (2004b). Medical interpreting and cross-cultural communication. Cambridge: Cambridge University Press.

Gile, D. (1995). Basic concepts and models for interpreter and translator training. Amsterdam \& Philadelphia: John Benjamins. Other. (n.d.). In Wikipedia. Retrieved from https://en.wikipedia.org/wiki/Other

Otherness. (n.d.). In Wikipedia. Retrieved from https://en.wikipedia.org/wiki/Otherness

REN, R. (2006). Analysis of understanding problems in intercultural language communication. Journal of Northeastern University (Social Science), 8(6), 446.

REN, R. (2009). On self and the other in language understanding. Journal of Northeastern University (Social Science), $11(2), 170$.

REN, R. (2010). On translator's visibility. Journal of Northeastern University (Social Science), 12(6), 540.

REN, R. (2012). Thingness: Falsifying the paradox of interpreter' s invisiblility. US-China Foreign Language, 10(3), 1025.

REN, R., LI, Y., \& WANG, M. (2012). On thingness of interpreters. Interpreting in the Age of Globalization-Proceedings of the 8th National Conference and International Forum on Interpreting, Chengdu, China.

REN, R. (Ed.). (2014). An Introduction to interpretation for graduates (Chinese ed.). Shenyang: Northeastern University Press. Wittgenstein, L. (1953). Philosophical investigations. New York: Macmillan. 